\title{
Intracardiac echocardiography for guidance of transcatheter tricuspid edge-to-edge repair
}

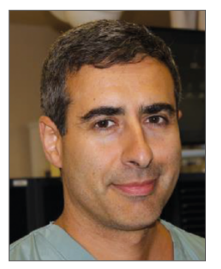

Neil P. Fam*, MD, MSc; Sondos Samargandy, MD; Sumeet Gandhi, MD; Janine Eckstein, MD

Division of Cardiology, St. Michael's Hospital, Toronto, Canada

This paper also includes supplementary data published online at: http://www.pcronline.com/eurointervention/142nd_issue/180

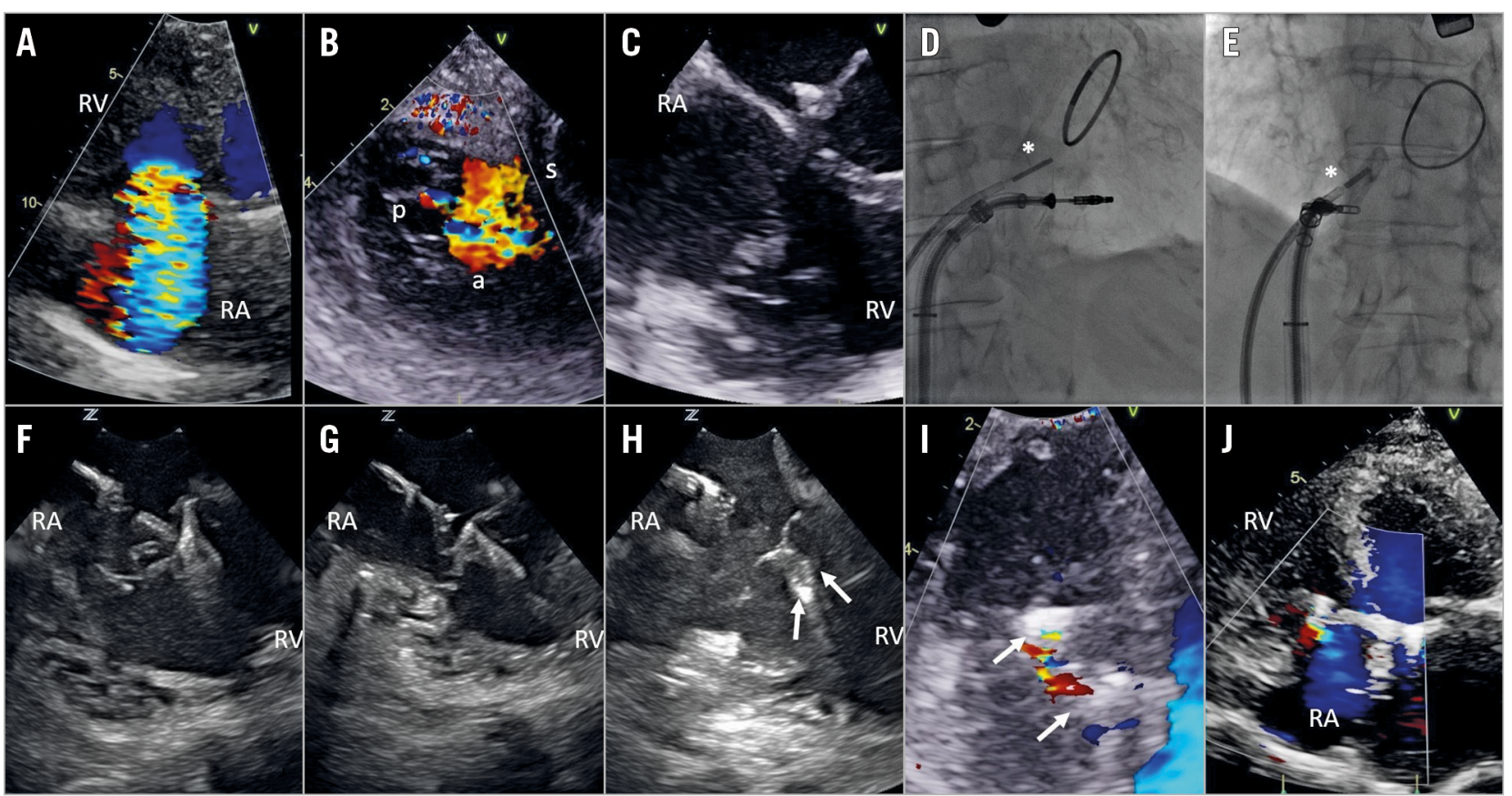

Figure 1. Intracardiac echocardiography for guidance of transcatheter tricuspid repair. A) Baseline TTE RV-focused view with colour Doppler demonstrating torrential TR. B) Baseline TEE transgastric view of tricuspid valve demonstrating torrential TR. C) Intraprocedural TEE demonstrating shadowing of tricuspid valve leaflets by mitral annuloplasty ring. D) Fluoroscopy in RAO projection of ICE catheter position (* indicates ICE catheter). E) Fluoroscopy in LAO projection of ICE catheter position (* indicates ICE catheter). F) Intraprocedural ICE view of MitraClip grasping of tricuspid valve posterior and septal leaflets. G) Intraprocedural ICE view of first MitraClip demonstrating tissue bridge. H) Intraprocedural ICE view following deployment of two MitraClips ( $\rightarrow$ indicates clips). I) Intraprocedural TEE transgastric view of tricuspid valve with simultaneous colour Doppler after implantation of two MitraClips ( $\rightarrow$ indicates clips). J) Post-procedural TTE apical four-chamber view demonstrating sustained TR reduction at one-month follow-up. a: anterior; ICE: intracardiac echo; p: posterior; RA: right atrium; RV: right ventricle; s: septal; TEE: transoesophageal echocardiography; TTE: transthoracic echocardiography

*Corresponding author: Division of Cardiology, St Michael's Hospital, 30 Bond St, Toronto, ON M5B 1W8, Canada. E-mail:famn@smh.ca 
A 73-year-old woman presented with NYHA Class III dyspnoea, fatigue and peripheral oedema. Past history included Barlow's disease with severe mitral regurgitation treated with a $32 \mathrm{~mm}$ Physio annuloplasty ring (Edwards Lifesciences, Irvine, CA, USA) and Alfieri stitch seven years previously, atrial fibrillation and chronic kidney disease. Echocardiography demonstrated massive tricuspid regurgitation (TR) due to tricuspid valve (TV) prolapse and annular dilatation (Figure 1A, Figure 1B, Moving image 1, Moving image 2). After Heart Team review, the patient was accepted for transcatheter tricuspid edge-to-edge repair with the MitraClip ${ }^{\circledR}$ (Abbott Vascular, Santa Clara, CA, USA).

Baseline transoesophageal echo (TEE) demonstrated significant shadowing of the TV leaflets by the mitral annuloplasty ring in multiple grasping views, precluding accurate assessment of septal leaflet insertion within the clip arms (Figure 1C, Moving image 3). Therefore, a 10 Fr ViewFlex ${ }^{\mathrm{TM}}$ Xtra intracardiac echo (ICE) probe (St. Jude Medical, St. Paul, MN, USA) was inserted via the left femoral vein for adjunctive procedural guidance. The ICE catheter was advanced into the right atrium, flexed anterior and leftward to a position just above the TV annulus, perpendicular to the clip arms and tricuspid leaflets providing an orthogonal longaxis grasping view (Figure 1D, Figure 1E). TEE imaging from the transgastric view was used to confirm perpendicularity of the clip arms to leaflet coaptation. The first clip was positioned between the posterior and septal leaflets, the second clip between the anterior and septal leaflets, reducing TR from massive to mild-moderate (Figure 1F-Figure 1I, Moving image 4-Moving image 8). At one-month follow-up, the patient reported remarkable symptomatic improvement with a reduction in diuretic requirements and sustained TR reduction (Figure 1J, Moving image 9).

Transcatheter tricuspid edge-to-edge repair is highly dependent on adequate echo imaging, typically with TEE. However, an important subset of patients may have suboptimal TEE imaging due to acoustic shadowing from intracardiac prostheses, lipomatous septum, or anatomic abnormalities such as kyphoscoliosis. ICE has been used for adjunctive procedural guidance of the MitraClip for mitral regurgitation after failed surgical annuloplasty $^{1}$, and recently as an alternative to TEE for percutaneous tricuspid annuloplasty ${ }^{2}$ and tricuspid edge-to-edge repair in a patient with previous oesophagectomy ${ }^{3}$. Here, we demonstrate the utility of tricuspid ICE imaging for edge-to-edge repair in a patient with suboptimal TEE imaging due to shadowing from a mitral annuloplasty ring. The inherent advantages of real-time ICE imaging for transcatheter tricuspid edge-to-edge repair include a steerable high-frequency transducer and high-resolution near-field imaging with the ability to position the ICE probe directly over the TV annulus in close proximity to the leaflets and clip arms, producing clear images for leaflet grasping. However, given that current ICE catheters lack multiplanar imaging, a complementary imaging modality such as fluoroscopy, TTE or TEE is needed for anatomi- cal reference. Larger studies comparing ICE and TEE-guided tricuspid intervention are warranted. As ICE technology evolves to provide true multiplanar and 3D imaging, there is the potential for truly minimally invasive tricuspid repair under local anaesthesia.

\section{Conflict of interest statement}

The authors have no conflicts of interest to declare.

\section{References}

1. Saji M, Rossi AM, Ailawadi G, Dent J, Ragosta M, Lim DS. Adjunctive intracardiac echocardiography imaging from the left ventricle to guide percutaneous mitral valve repair with the MitraClip in patients with failed prior surgical rings. Catheter Cardiovasc Interv. 2016;87:E75-82.

2. Latib A, Mangieri A, Vicentini L, Ferri L, Montorfano M, Ismeno G, Regazzoli D, Ancona MB, Giglio M, Denti P, Colombo A, Agricola E. Percutaneous Tricuspid Valve Annuloplasty Under Conscious Sedation (With Only Fluoroscopic and Intracardiac Echocardiography Monitoring). JACC Cardiovasc Interv. 2017; 10:620-1.

3. Pozzoli A, Taramasso M, Zuber M, Maisano F. Transcatheter tricuspid valve repair with the MitraClip system using intracardiac echocardiography: proof of concept. EuroIntervention. 2017; 13:e1452-3.

\section{Supplementary data}

Moving image 1. Preprocedural TTE RV-focused apical view with colour Doppler demonstrating torrential TR.

Moving image 2. Preprocedural TEE transgastric view of the tricuspid valve with simultaneous colour Doppler demonstrating torrential TR.

Moving image 3. Preprocedural TTE demonstrating shadowing of TV leaflets from mitral annuloplasty ring.

Moving image 4. Intraprocedural ICE of grasping TV posterior and septal leaflets.

Moving image 5. Intraprocedural ICE of first closed MitraClip with tissue bridge.

Moving image 6. Intraprocedural ICE of second MitraClip dropping of grippers on anterior and septal leaflets.

Moving image 7. Intraprocedural ICE following deployment of two MitraClips.

Moving image 8. Intraprocedural TEE transgastric view of tricuspid valve with simultaneous colour Doppler after implantation of two MitraClips.

Moving image 9. Post-procedural TTE apical four-chamber view demonstrating sustained reduction in TR at one-month follow-up.

The supplementary data are published online at:

http://www.pcronline.com/

eurointervention/142nd issue/180 